\title{
A Note on Portunid Crabs of Odisha Coast, India
}

\author{
Dev Roy $\mathrm{MK}^{1}$, Rath $\mathrm{S}^{2}$ and Nandi $\mathrm{NC}^{1 *}$ \\ ${ }^{1}$ Social Environmental and Biological Association, India \\ ${ }^{2}$ Zoological Survey of India, India
}

Submission: February 09, 2017; Published: February 13, 2017

*Corresponding author: Nandi NC, Social Environmental and Biological Association, Kolkata, India, Tel: 9674002604; Email: nepalchandra.nandi@gmail.com

\section{Opinion}

Portunid crabs belonging to the genera, viz., Scylla, Portunus and Charybdis species mostly represent edible shellfishes with high quality meat content. These crabs are widely valued in domestic and export markets Nandi \& Pramanik [1]. They are mostly marine species; estuarine forms are also not uncommon. In the eastern maritime States of India, Odisha coast is $480 \mathrm{~km}$ long with a continental shelf area of 32,000 sq km. In this coastal region there are a number of estuaries (Mahanadi, Rhushikulya, Bahuda, Brahmani-Baitarini) and a large brackish water lagoon, known as Chilka Lake, an important Ramsar site in India. All these coastal habitats support important fish and shell fish resources of the country. A perusal of literature as well as investigation undertaken by the authors reveals a total of 29 portunid crabs species belonging to three subfamilies and six genera (Table 1) from this coast including five new records, (Table 2, Figure 1) viz., Portunus (Xiphonectes) hastatoides (Fabricius), Charybdis (Charybdis) lucifera (Fabricius), Charybdis (Charybdis) variegata (Fabricius) and Charybdis (Goniohellenus) truncata (Fabricius). It is mentioned that literature studies have revealed that in India, Andaman and Nicobar Islands support maximum number of 55 portunid species Dev Roy \& Nandi [2], followed by 53 species from Tamil Nadu coast Dev Roy \& Nandi [3] 23 species from West Bengal coast Dev Roy \& Nandi [4] and 22 species from Andhra Pradesh coast Dev Roy \& Nandi [5]. Among the species listed, Scylla serrata, S. olivacea, S. tranquebarica, Portunus pelagicus and $P$. sanguinolentus are highly exploited from this coast. Thus, it is necessary to encourage culture of crabs, especially mud crab fattening and farming segregating mud crab species towards cost effective production of crabs Nandi, et al. [6], Pramanik \& Nandi [7] and to meet the local demand as well as export market.

Table 1: List of Portunid crabs of Odisha coast.

\begin{tabular}{|c|c|}
\hline & Family Portunidae Rafinesque, 1815 \\
\hline 1 & Subfamily Caphyrinae Paul'son, 1875 \\
\hline & Lissocarcinus polybioides Adams and White, 1848 \\
\hline 2 & Subfamily Podophthalminae Dana, 1851 \\
\hline & Podophthalmus vigil (Fabricius, 1798) \\
\hline 3 & Subfamily Portuninae Rafinesque, 1815 \\
\hline 4 & Portunus (Monomia) argentatus (White, 1847) \\
\hline 5 & Portunus (Monomia) gladiator Fabricius, 1798 \\
\hline 6 & Portunus (Monomia) gracilimanus (Stimpson, 1858) \\
\hline 7 & Portunus (Portunus) pelagicus (Linnaeus, 1758) \\
\hline 8 & Portunus (Portunus) sanguinolentus (Herbst, 1803) \\
\hline 9 & *Portunus (Xiphonectes) hastatoides (Fabricius,1798) \\
\hline 10 & Scylla serrata (Forskål, 1775) \\
\hline & Scylla olivacea (Herbst, 1796) \\
\hline
\end{tabular}


Fisheries and Oceanography Open Access Journal

\begin{tabular}{|c|c|}
\hline 11 & Scylla tranquebarica (Fabricius, 1798) \\
\hline & Subfamily Thalamitinae Paul'son, 1875 \\
\hline 12 & Charybdis (Charybdis) affinis Dana, 1852 \\
\hline 13 & ${ }^{*}$ Charybdis (Charybdis) annulata (Fabricius, 1798) \\
\hline 14 & Charybdis (Charybdis) amboinensis Leene and Buitendijk, 1938 \\
\hline 15 & Charybdis (Charybdis) callianassa (Herbst, 1801) \\
\hline 16 & Charybdis (Charybdis) feriata (Linnaeus, 1758) \\
\hline 17 & Charybdis (Charybdis) helleri (A. Milne Edwards, 1861) \\
\hline 18 & ${ }^{*}$ Charybdis (Charybdis) lucifera (Fabricius, 1798) \\
\hline 19 & Charybdis (Charybdis) miles (De Haan, 1835) \\
\hline 20 & Charybdis (Charybdis) natator (Herbst, 1794) \\
\hline 21 & Charybdis (Charybdis) rivers andersoni Alcock, 1899 \\
\hline 22 & Charybdis (Charybdis) rostrata A Milne Edwards, 1861 \\
\hline 23 & ${ }^{*}$ Charybdis (Charybdis) variegata (Fabricius, 1798) \\
\hline 24 & Charybdis (Goniohellenus) hoplites (Wood-Mason, 1877) \\
\hline 25 & ${ }^{*}$ Charybdis (Goniohellenus) truncata (Fabricius, 1798) \\
\hline 26 & Charybdis (Goniohellenus) vadorum Alcock, 1899 \\
\hline 27 & Charybdis (Gonioneptunus) bimaculata (Miers, 1886) \\
\hline 28 & Thalamita crenata (Latreille, 1829) \\
\hline 29 & Thalamita imparimana Alcock, 1899 \\
\hline
\end{tabular}

Table 2: New records of portunid crab species with locations and geographical coordinates.

\begin{tabular}{|c|c|c|}
\hline Name of Species & Lat./Long. & $\begin{array}{c}\text { Name of } \\
\text { Place }\end{array}$ \\
\hline $\begin{array}{c}\text { Charybdis(Charybdis) } \\
\text { lucifera (Fabricius,1798) }\end{array}$ & $\begin{array}{c}20.3166^{\circ} \mathrm{N}, 86.9640^{\circ} \mathrm{E} \\
19.2647^{\circ} \mathrm{N}, 84.8620^{\circ} \mathrm{E}\end{array}$ & $\begin{array}{c}\text { Dhamra } \\
\text { Gopalpur }\end{array}$ \\
\hline $\begin{array}{c}\text { Charybdis(Charybdis) } \\
\text { variegata (Fabricius,1798) }\end{array}$ & $20.3166^{\circ} \mathrm{N}, 86.6114^{\circ} \mathrm{E}$ & Paradeep \\
\hline $\begin{array}{c}\text { Charybdis(Goniohellenus) } \\
\text { truncata (Fabricius,1798) }\end{array}$ & $\begin{array}{c}20.3166^{\circ} \mathrm{N}, 86.9640^{\circ} \mathrm{E} \\
19.2647^{\circ} \mathrm{N}, 84.8620^{\circ} \mathrm{E}\end{array}$ & $\begin{array}{c}\text { Dhamra } \\
\text { Gopalpur }\end{array}$ \\
\hline $\begin{array}{c}\text { Portunus(Xiphonectes) } \\
\text { hastoides(Fabricius, 1798) }\end{array}$ & $20.3166^{\circ} \mathrm{N}, 86.9640^{\circ} \mathrm{E}$ & Dhamra \\
\hline
\end{tabular}

\section{References}

1. Nandi NC, Pramanik SK (1994) Crabs and Crab Fisheries of Sundarban. Hindustan Publishing Corporation, Delhi, India, pp. 1-192.

2. Dev Roy MK, Nandi NC (2012) Brachyuran crabs (Crustacea). Fauna of Andaman and Nicobar Islands, State Fauna Series. Zool Surv, Kolkata, India 19(1): 1-236.

3. Dev Roy MK, Nandi NC (2007a) Brachyuran diversity in coastal ecosystems of Tamil Nadu. J Environ \& Sociobiol 4(2): 169-192.

4. Dev Roy MK, Nandi NC (2008) Checklist and distribution of brachyuran crabs of West Bengal. J Environ \& Sociobiol 5(2): 193-216.

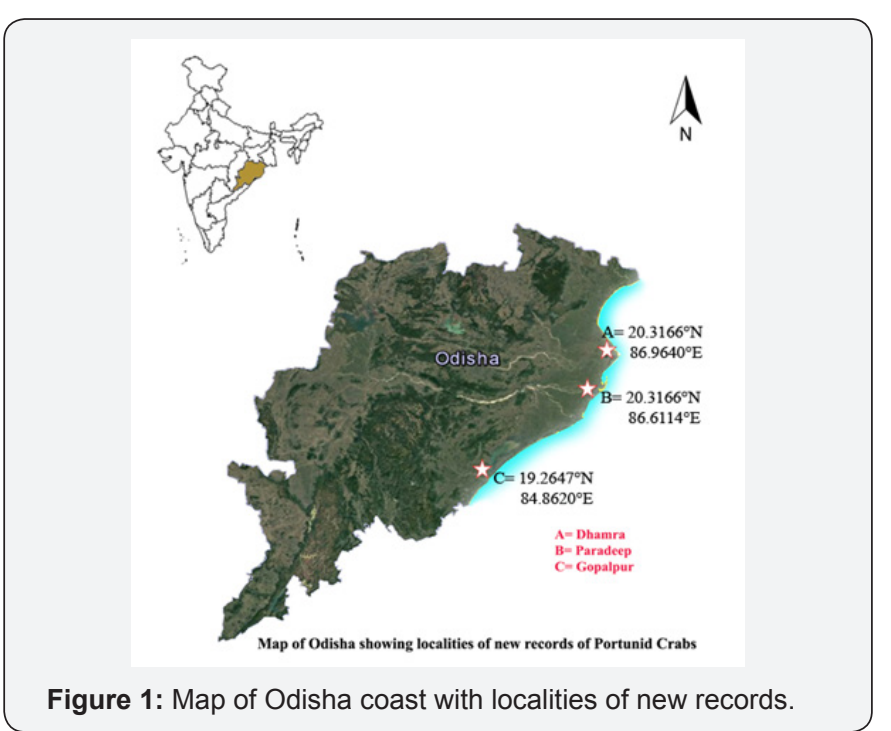

5. Dev Roy MK, Nandi NC (2007b) Brachyuran bioresources of coastal Andhra Pradesh. In: National Symposium on Conservation and Valuation of Marine Biodiversity, Zool Surv Kolkata, India, pp 1-445.

6. Nandi NC, Pramanik SK, Dev Roy MK (2016) Mud Crab Culture: Relevance of Species Identity in Production Economics with Reference to Sundarban Coast. J Fisheries Sciences 10(4): 84-89.

7. Pramanik CSK, Nandi NC (2012) Crab fattening (Chamber chas)A promising enterprise in Indian part of Sundarban. J Environ \& Sociobiol 9(1): 78. 
(C) This work is licensed under Creative

\section{Your next submission with Juniper Publishers} will reach you the below assets

- Quality Editorial service

- Swift Peer Review

- Reprints availability

- E-prints Service

- Manuscript Podcast for convenient understanding

- Global attainment for your research

- Manuscript accessibility in different formats ( Pdf, E-pub, Full Text, Audio)

- Unceasing customer service

Track the below URL for one-step submission https://juniperpublishers.com/online-submission.php 